\title{
The Molecular Aspects of Personalized Anticancer Treatment
}

\author{
N. Cherdyntseva ${ }^{1,2, a)}$, N. Litviakov ${ }^{1,2, b)}$, F. Ivanova ${ }^{3, c)}$, E. Denisov ${ }^{1,2,4, d)}$, \\ P. Gervas ${ }^{1, e)}$, and E. Cherdyntsev ${ }^{4, f)}$ \\ ${ }^{I}$ Tomsk Cancer Research Institute, Tomsk, 634050 Russia \\ ${ }^{2}$ Laboratory for Translational Cellular and Molecular Biomedicine, Tomsk State University, \\ Tomsk, 634050 Russia \\ ${ }^{3}$ Yakutsk Oncology Dispensary, Sacha Republic, Russia \\ ${ }^{4}$ Tomsk Polytechnic University, Tomsk, 634050 Russia

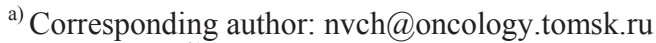 \\ b) nvlitv72@yandex.ru \\ ${ }^{c)}$ Feodossia iv@inbox.ru \\ d) d_evgeniy@oncology.tomsk.ru \\ e) gervaspa@oncology.tomsk.ru \\ f) es@tpu.ru
}

\begin{abstract}
Only 25\% of cancer patients, on average, benefit from therapy. Even in the cases of complete clinical response the tumor progression is an event of high level expectation. The main reasons for tumor progression are: intratumor heterogeneity resulted from clonal evolution, drug resistance, and tumor-promoting microenvironment. The reprogramming of microenvironmental stromal-inflammatory components is expected to allow tumor phenotype reversion. So, to find the new effective markers of tumor progression, drug response and targets for therapy, it could be promising to take into account the tumor-microenvironment heterogeneity and tumor clonal evolution.
\end{abstract}

\section{TUMOR CLONAL EVOLUTION REQUIRES THE PERSONALIZED THERAPY FOR CANCER PATIENTS}

The main types of treatment for cancer are: surgical removal of the primary tumor and metastasis, radiotherapy and chemotherapy, including targeted therapy directing to dominant tumor specific molecular aberration. Cancer is known to be a multifactorial pathology developing under the influence of carcinogenic factors, life style, including diet and reproductive behavior, the effects of which are very variable in different individuals due to the unique genetic characteristics for each person.

The complexity of tissue ecosystem of cancer cells is endowed by the interrelationship between exposures to environmental agents of chemical, physical and biological nature, the constitutive genetics of the host cells, systemic regulators, local regulators (microenvironment) and architectural constraints, which provide the evolution of somatic cells, tumor biological behavior and finally the disease outcome. Each person is undoubtedly a unique ecosystem that requires the individual (personalized) approaches to the treatment of cancer. The question "Who or what should be treated: the patient or disease?" has the only answer: "The patient should be treated!!!". This is the basis for the development of personalized medicine. Personalized medicine means the application of patient-specific genetic information (both germline and somatic) and molecular and/or cellular tumor characteristics to select the optimal treatment for individual patient with the goal of improved therapeutic efficacy and reduced toxicity [1].

In accordance with Peter Nowell ("Clonal evolution of tumor cell population", Science, 1976), the tumor evolutionary trajectories are complex and branching, in parallel with Darwin's iconic evolutionary speciation tree. 
Cancer appears to be a process of a clonal evolution where sequential acquisition of mutations with concomitant, successive subclonal dominance or selective sweeps result in the tumors with various molecular aberrations that requires personalized approach to treatment [2].

Tumor clonal evolution provides intratumor heterogeneity which is the coexistence of different types of tumor clones having specific biological features providing different potential to contribute to tumor progression and variability in response to chemotherapy [3]. Tumor behavior in each patient is endowing by specific moleculargenetic aberrant peculiarities of tumor cells and microenvironment. We performed the microarray analysis to measure the expression of various genes in each tumor structure using laser microdissection, thus avoiding the interference of cells from the other compartment. We revealed that trabecular structures demonstrated the activity of the genes coding for $\mathrm{ABC}$ transporters more often than the other morphological variants. The marked variation in the spectrum as well as in functionality of main signal pathways in different tumor structures point to their different contribution to tumor progression, which could be useful to identify new prognostic markers and molecular targets to the therapeutic intervention. These results indicate that intratumoral morphological heterogeneity in breast cancer correlates with expression profile of drug resistance genes reflecting different patterns of responsiveness to chemotherapy [4-6].

Chemotherapy may destroy cancer clones and erode their habitats. But it can also provide a potent selective pressure for the expansion of resistant variants replacing the dead tumor cells.

\section{CONVENTIONAL CHEMOTHERAPY}

Chemotherapy (CT) as a cancer treatment modality has been developed and introduced into clinical practice for more than 70 years and has demonstrated the realistic benefit for cancer patients' survival. Molecular mechanisms of CT clinical effectiveness involve different cell signaling pathways such as drug methabolism, transmembrane transport, interaction with target, apoptosis and DNA reparation. Variations in the activity of these processes in different patients due to innate genetic variabilities result in the different clinical responses to drug.

Despite the fast development of target-directing chemotherapy, standard (conventional) drug treatment is now widely required all over the world. Unfortunately, the clinical response to CT has not been detected in 40-60\% of cancer patients [7]. The number of molecular markers has been discovered to predict the tumor response to various drugs in each individual, but clinical benefit cannot be completely determined.

Marker detection before treatment to choose suitable drug for each patient could results in the increase in CT efficacy [8].

Such conventional drugs as irinotecan, vinorelbine, antracyclines, cisplatin, capecitabine, gemzar, taxanes are prevalent in anticancer therapy all over the world. The markers, which need to be tested for choosing the treatment algorithm, are as follows: TOP 1, TUBB2, TUBB3, TOP2, TYMS, TYMP, ERCC1, BRCA1 cyclin D [9].

We showed that personalized cancer treatment resulted in the benefit in metastases-free survival within 40 months of follow-up in breast cancer patients treated with various regimens of preoperative therapy.

\section{CONVENTIONAL CHEMOTHERAPY AND ETHNICITY}

Ethnic differences in drug susceptibility and toxicity have been demonstrated by different investigators. These differences may be attributed, at least in part, to population-related differences in the frequency of DNA polymorphisms as inherited variations at the DNA sequence level, contributing to variability of different pharmacokinetics [10].

There is substantial interindividual variability in the efficacy and tolerability of anticancer drugs. Such differences can be even greater between individuals of different ethnicities. The differences in pharmacodynamics which really display the drug efficacy depend on environmental factors, live style (smoking, Herbal, diet, etc.), some genetic factors (polymorphisms, mutations) [10]. The large multicenter clinical investigation recruiting white American and Japanese patients with the same type of cancer showed that identical treatment with chemotherapy led to the different clinical results including both the tumor response and the systemic toxicity [10].

Our study showed a substantial difference in their reaction to platinum drugs between Asians and Caucasians. To determine whether population-related genomics contribute to differences in patient outcomes, pharmacogenomic relevance of polymorphisms in some genes, involved in cisplatin metabolism, were examined. Nineteen polymorphisms in ten genes were tested for correlations with the efficacy and toxicity of a cisplatincyclophosphamide regimen in Yakut and Russian patients with ovarian cancer. The CYP2E1 7632T >A 
polymorphism was associated with progression-free survival $(p=0.015)$ in the Yakuts. In Russians, progression-free survival was correlated with the GSTP1 Ile105Val polymorphism $(p=0.004)$. Yakut patients with the GSTT1-null genotype had a higher risk for nephrotoxicity. Nephrotoxicity in Russian groups was more frequent among the patients with heterozygous ERCC1 genotypes. Severe emesis in the Yakuts was independently associated with two polymorphisms in the CYP2E1 gene but in Russians, it was more common in patients with the GSTT1-null genotype. Thus, significant differences in genotype distribution between Russian and Yakut women were observed for ten of the 19 polymorphisms, but none of them seemed to be a clear casual candidate and further studies involving more markers are required [11].

The development of approaches to treatment personalization to increase efficacy and decrease toxicity can use the testing of inherited BRCA 1/2 mutation, because the carriers of mutated allele have dramatically increased sensitivity to cisplatin. According to other authors' data there are ethnic differences in a mutation type between populations. Ethnic Aspects of Hereditary Breast Cancer in the Region of Siberia have been studied in our Institute. Ethnic diversity of the population in the region of Siberia suggests the existence of different germline mutations in the BRCA1/2 genes associated with breast and ovarian cancers in different ethnic populations. Our aim was to evaluate the frequency of the most common mutations, BRCA1/2 (BRCA1 5382insC, BRCA1 185delAG, BRCA1 4153delAG, BRCA1 T300G, BRCA2 6174delT) in women diagnosed with breast cancer among indigenous people and newcomers living in Siberia. We tested 1281 genomic DNA samples for the presence of BRCA1 5382insC mutation in patients diagnosed with breast cancer considering no family history. Out of 765 patients of Slavic ethnic group, 27 women $(3.5 \%)$ were carriers of allele BRCA1 5382insC. The frequencies of mutations in patients with signs of hereditary cancer were: $8.3 \%$ in the group of young patients (under 40 years), $20.0 \%$ in the patients with bilateral cancer and $5.7 \%$ in the patients with family history of breast or ovarian cancers. We tested 516 BC patients residing on the territory of the Buryat-Aginsky district, Republics of Tyva and Altai. Out of them, there were 197 patients among the indigenous population (buryats, tuvinians, altaians), and 319 patients among newcomers (Slavic ethnics). BRCA1 5382insC mutations were detected only in women from Slavic ethnic groups. BRCA1 $5382 \mathrm{insC}$ allele was not found in indigenous breast cancer patients, although 59 patients had signs of hereditary cancer. In the women from Slavic ethnic group, the BRCA1 185delAG, BRCA1 4153delAG and BRCA1 T300G mutations were detected in $9.1 \%$ of cases and were not found in the patients among the indigenous population [12]. Thus, our study results indicate the advisability of further studies to identify the genes responsible for the occurrence of hereditary breast cancer in the indigenous population.

\section{MULTIDRUG RESISTANCE}

Multidrug resistance (MDR) is one of the crucial reasons for chemotherapy (CT) failure in cancer therapy. ABCtransporters are responsible for the active efflux of multitude cytotoxic compounds across cell membrane abrogaiting the chemotherapy efficacy. $\mathrm{CT}$ is known to induce drug resistance by activation of $\mathrm{ABC}$ gene expression and there is not any effective drug to inhibit $\mathrm{ABC}$ protein activity because the therapeutic dose shows very high toxicity. We have found that the decrease in $\mathrm{ABC}$-transporter expression during the neoadjuvant treatment relates to drug response. In contrast, the increase in the $\mathrm{ABC}$ gene expression was associated with no clinical response to treatment [13].

In the previous study we performed a combined analysis of MDR gene locus deletions in tumor DNA, MDR gene expression and clinical response to NAC in breast cancer patients. Copy number variations (CNVs) in biopsy specimens were tested using high-density microarray platform CytoScanTM HD Array (Affymetrix, USA). 75$100 \%$ persons having deletions of MDR gene loci demonstrated the down-regulation of MDR gene expression. Expression of MDR genes was 2-8 times lower in the patients with deletion than in the patients having no deletion only in post-NAC tumors samples, but not in tumor tissue before chemotherapy. All patients with deletions of ABCB1 ABCB3 ABCC5 gene loci, and most patients having deletions in ABCC1 (16p13.1), ABCC2 (10q24), ABCG1 (21q22.3), ABCG2 (4q22.1), responded favorably to NAC. So, our study demonstrated that deletion MDR gene loci can be used as predictive marker for tumor response to NAC $[13,14]$. We performed the prospect study to examine whether the detection of new markers for prognosis of tumor response can be useful to choose the optimal therapy. Personalized preoperative chemotherapy in accordance with appropriate marker test provided the clinical response in $86 \%$ of the patients while the individuals receiving standard treatment showed the tumor response only in $45 \%$ of cases. 


\section{TARGETED THERAPY}

One of the novel approaches to cancer therapy is a so called targeted therapy where drug acts on specific target (genetic or molecular alteration) in tumor cells to kill it or stop its proliferation. Targeted drugs are designed to interfere with specific molecules necessary for tumor growth and progression. Two types of drugs have been developed: specific monoclonal antibodies inhibiting the signal from amplified receptor necessary for tumor growth and progression, and tyrosine-kinase receptor inhibitors interfering with tumor promoting signaling pathway, activated by mutation in the receptor of tyrosine-kinase. A lot of different molecular targets in tumor cells, related to specific promalignant cellular signaling pathways are identified, for example in lung cancer, including such molecules as EGFR (epidermal growth factor receptor), BRAF, ROS, PDGFR, MET etc. The following specific drugs have been designed: Vemurofenib, lapatinib, sunitinib, krizotinib, erlotinib, AZD6244, BEZ235, which are currently under investigation in clinical trials.

Targeted therapy appeared to be effective in $80-90 \%$ of patients, whose tumors bear specific molecular alterations. For example, tyrosine-kinase receptor inhibitor Gefitinib (Iressa) results in tumor response in $50-70 \%$ of cases and the average time to progression of 21.7 months versus $40 \%$ tumor response and 5.5 month time to progression in standard CT. The next prevalent targeting drug is the monoclonal antibody (Trasuzumab or Herceptin) blocking the HER2 (receptor for epidermal growth factor 2) signaling. Tumor Response is increased to $47.3 \%$ versus $34.5 \%$ in the standard CT. One of the targeted therapy problems is multidrug resistance. Another problem is an intratumor heterogeneity, denominated as coexistence within the same tumor of the cell populations with various biological behavior and different genetic aberrations. Chemotherapy is the strong factor promoting the clonal evolution of tumor. This tumor provides the alteration in genetic landscape and emergence of drug resistant tumor clones. The disappearing of few tumor clones after preoperative therapy as well as the simultaneous origin of new tumor clones has been shown in our study.

The choosing of a specific targeted drug to each patient is impossible now due to the lack of knowledge about all driver mutations (as molecular targets) for various cancer types. Signaling pathway parallelism can result in the failure of therapeutic effect of one targeted drug because of the activation of another tumor promoting signal transduction.

Tumor progression: relapses and metastasis are frequent events following targeted therapy. What is the perspective to avoid it? One of the suitable opportunities is the development of precision medicine to overcome tumor heterogeneity. It requires monitoring tumor evolution to identify dominant clones for choosing targeted drug which is needed for effective therapy.

So, modern clinical investigation seems to be performed using the following steps: tumor tissue (biopsy)-tumor genome discovery-IHC (immunohistochemistry) in situ-genome wide sequencing-microarray analysis-detection of driver mutations and molecular aberrations-target drug selection for treatment. Primary tumor is not enough to provide biopsies for many times on different steps of treatment monitoring, that dictates a necessity of having liquid biopsy for tumor genome monitoring as an alternative way.

Genomic characteristics of circulating tumor cells as well as circulating tumor-specific DNA allow searching for driver mutations providing CT resistance and dominant clone identification to choose target drug.

The problem is to find driver (dominant) mutations which are responsible to tumor progression and to validate their clinical significance.

\section{TUMOR AND MICROENVIRONMENT}

The theory of somatic mutation considers the cancer as a pathological process induced by the genome deregulation in a single somatic cell, and the main therapy goal seems to be to remove or kill cancer cells. Tissue organisation field theory declares cancer arising as a result of dysregulation between cells and their microenvironment, leading to compromising genomic integrity and considers cancer as curable and reversible process $[15,16]$. According to the hypothesis "Seed and Soil" [17] and the conception of metastatic niches [18] metastasis efficacy is provided not only by the tumor cells characteristics but also the microenvironment features in primary tumors and secondary sites for metastases (metastatic niches).

The study of molecular mechanisms of cancer metastasis based on Lyden's metastatic niche concept and Paget "seed and soil" hypothesis could be a scientific platform for prediction and prevention of metastatic disease.

Novel approaches to treatment of metastatic disease presume that anticancer therapy must be directed not only to tumor cell elimination, but also to microenvironment regulation and metastatic niches treatment to reverse tumor 
phenotype or/and to damage of pre-metastatic niche formation. Re-education of stromal and inflammatory cells, as well as another microenvironmental component rather than targeted ablation per se, may be an effective strategy for cancer treatment.

\section{ACKNOWLEDGMENTS}

The study was supported by a grant from the Russian Science Foundation No. 14-15-00318 (review of the authors' own data) and the Tomsk State University Competitiveness Improvement Program.

The study reported in this article was conducted according to accepted ethical guidelines involving research in humans and/or animals and was approved by an appropriate institution or national research organization.

The study is compliant with the ethical standards as currently outlined in the Declaration of Helsinki.

All individual participants discussed in this study, or for whom any identifying information or image has been presented, have freely given their informed written consent for such information and/or image to be included in the published article.

\section{REFERENCES}

1. ESMO Handbook of Translational Research, edited by G. Tortora, C. Sessa, A. Scarpa, and S. Banerjee (ESMO Press, 2015).

2. P. C. Nowell, Science 194(4260), 23-28 (1976).

3. M. Greaves and C. C. Maley, Nature 481(7381), 306-313 (2012)

4. M. V. Zavyalova, V. M. Perelmuter, S. V. Vtorushin, E. V. Denisov, N. V. Litvyakov, E. M. Slonimskaya, and N. V. Cherdyntseva, Diagnostic Cytopathology 41(3), 279-282 (2013).

5. E. V. Denisov, T. S. Gerashchenko, M. V. Zavyalova, N. V. Litviakov, M. M. Tsyganov, E. V. Kaigorodova, E. M. Slonimskaya, J. Kzhyshkowska, N. V. Cherdyntseva, and V. M. Perelmuter, Neoplasma 62(3), 405-411 (2015).

6. E. V. Denisov, N. V. Litviakov, M. V. Zavyalova, V. M. Perelmuter, S. V. Vtorushin, M. M. Tsyganov, T. S. Gerashchenko, E. Y. Garbukov, E. M. Slonimskaya, and N. V. Cherdyntseva, Sci. Rep. 4, 4709 (2014).

7. A. Berruti, M. P. Brizzi, D. Generali, M. Ardine, L. Dogliotti, P. Bruzzi, and A. Bottini, Oncologist 13(11), 1137-1148 (2008).

8. R. Gao, D. K. Price, T. Sissung, E. Reed, and W. D. Figg, Molecular Cancer Therapeutics 7(5), 1246-1250 (2008).

9. M. Tanner, J. Isola, T. Wiklund, B. Erikstein, P. Kellokumpu-Lehtinen, P. Malmstrom, N. Wilking, J. Nilsson, and J. Bergh, J. Clinical Oncology 24(16), 2428-2436 (2006).

10. P. H. O’Donnell and M. E. Dolan, Clinical Cancer Res. 15, 4806-4814 (2009).

11. A. Khrunin, F. Ivanova, A. Moisseev, D. Khokhrin, Y. Sleptsova, V. Gorbunova, and S. Limborska, Pharmacogenomics 13(2), 171-178 (2012).

12. N. V. Cherdyntseva, L. F. Pisareva, A. A. Ivanova, Y. V. Panferova, E. A. Malinovskaya, I. N. Odintsova, A. V. Doroshenko, P. A. Gervas, E. M. Slonimskaya, A. A. Shivitool, V. V. Dvornichenko, and Y. L. Choinzonov, Vestnik Ros. Akad. Med. Nauk 11-12, 72-79 (2014).

13. N. V. Litviakov, N. V. Cherdyntseva, M. M. Tsyganov, et al., Cancer Chemother Pharmacol 71(1), 153-163 (2013).

14. N. V. Litviakov, N. V. Cherdyntseva, M. M. Tsyganov, et al., Oncotarget 7(7), 7829-7841 (2016).

15. D. Hanahan and R. A. Weinberg, Cell 144(5), 646-674 (2011).

16. A. M. Soto, C. Sonnenschein, P. K. Maini, and D. Noble, Progress Biophysics Molecular Biology 106(2), $337-$ 339 (2011).

17. S. Paget, Cancer Metastasis Rev. 8, 98-101 (1989).

18. D. Lyden, K. Hattori, S. Dias, et al., Nat. Med. 7, 1194-1201 (2001). 